\title{
Synthesis of Stereodefined Unsaturated Acyclic Fragments
}

\section{Precursor synthesis:}

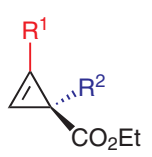

1. $\mathrm{R}^{3} \mathrm{MgBr}$ (1.2 equiv), Cul (20 $\left.\mathrm{mol} \%\right), \mathrm{Et}_{2} \mathrm{O},-35^{\circ} \mathrm{C}$

2. $\mathrm{ZnBr}_{2}$ (1.5 equiv, $1 \mathrm{M}$ in THF), -50 to $-20^{\circ} \mathrm{C}$

3. reagent (1.2-3.0 equiv), $\left[\mathrm{Pd}\left(\mathrm{PPh}_{3}\right)_{4}\right](5 \mathrm{~mol} \%)$, THF or DMF, $45-75^{\circ} \mathrm{C}$

4. $\mathrm{LiAlH}_{4}$ or DIBAL (2.5 equiv)

$$
\mathrm{R}^{1}=\text { Alk }
$$

$\mathrm{R}^{2}=\mathrm{H}, \mathrm{D}, \mathrm{Me}, \mathrm{Ph}$

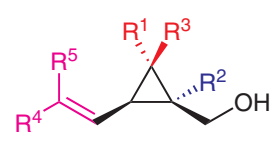

$22-82 \%$ yield

22 examples gram scale synthesis

dr > 95:5:0:0 either $E$ or $Z$ olefin

Oxidative palladium-catalyzed Heck coupling:

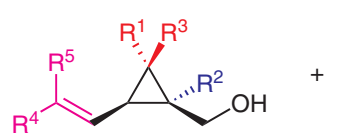

$$
\begin{gathered}
\underset{\left.\mathrm{Pd}(\mathrm{OTs})_{2}(\mathrm{MeCN})_{2}\right](7.5 \mathrm{~mol} \%)}{\text { ligand }(9 \mathrm{~mol} \%)} \\
\mathrm{Cu}(\mathrm{OTf})_{2}(5 \mathrm{~mol} \%)
\end{gathered}
$$

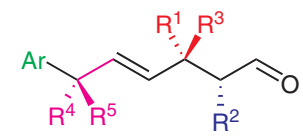

(3.0 equiv)

Selected examples:<smiles>C[C@H](/C=C/[C@H](C)c1ccccc1)CC=O</smiles>

$78 \%$ yield from $(Z)$ olefin dr $>$ 95:5<smiles>CCCC[C@](C)(/C=C/[C@@H](C)c1ccc(OC)c(OC)c1)CC=O</smiles>

$70 \%$ yield from $(Z)$ olefin $\mathrm{dr}>95: 5$

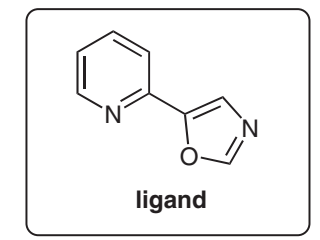

ligand

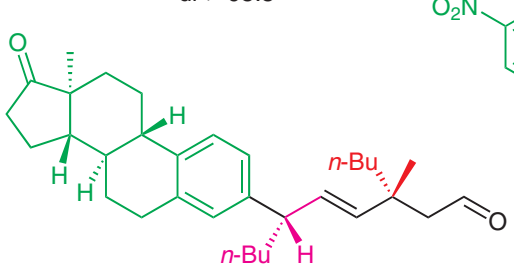<smiles>COCc1ccc(C)cc1</smiles>

$51 \%$ yield

from $(E)$ olefin

dr > 95:5 (acyclic stereocenters)

$40 \%$ yield from $(E)$ olefin $\mathrm{dr}>95: 5$

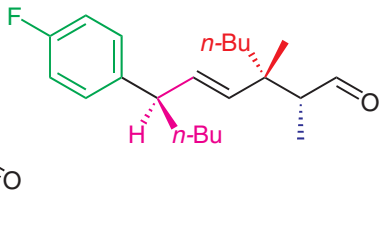

$54 \%$ yield from $(Z)$ olefin $\mathrm{dr}=86: 14: 0: 0$
Significance: Marek and co-workers report a highly stereodivergent route to unsaturated acyclic fragments in good to excellent yields. The key step for this transformation is the oxidative palladiumcatalyzed Heck coupling of aryl boronic acids with alkenylcyclopropyl carbinols.

SYNFAcTs Contributors: Paul Knochel, Moritz Balkenhohl

Synfacts 2018, 14(11), 1175 Published online: 18.10.2018 DOI: 10.1055/s-0037-1611035; Reg-No.: P13018SF
Comment: Remarkably, by using this method, all four stereoisomers of similar scaffolds can be synthesized. Furthermore, in addition to various arylboronic acids, alkenyl triflates or heteroarylboronic acids can be employed in the Heck reaction. 